\title{
Cutibacterium acnes endocarditis of Prosthetic Valves: A case series
}

\author{
Mark Boyle ${ }^{1}$, Charlene Tennyson ${ }^{1}$, Achyut Guleri ${ }^{1}$, and Antony Walker ${ }^{1}$ \\ ${ }^{1}$ Blackpool Victoria Hospital
}

March 6, 2021

\begin{abstract}
Cutibacterium acnes (C. acnes), previously known as Propionibacterium acnes (although likely underreported), is a rare cause of infective endocarditis (IE) and often difficult to diagnose. We describe three cases from a single centre over a five-year period to provide insight into the various clinical presentations, progression and management of patients with this infection. The primary objective of our series is to highlight the difficulty in the initial assessment of these patients with an aim to improve the time and accuracy of diagnosis and expedite subsequent treatment. There are currently no guidelines in the literature for the management of IE caused by Cutibacterium acnes. Our secondary objectives are to disseminate information about the indolent course of the disease and add to the growing body of evidence around this rare yet complex cause of IE.
\end{abstract}

\section{Hosted file}

C ACNES paper - JOC revision.pdf available at https://authorea.com/users/399954/articles/ 512350-cutibacterium-acnes-endocarditis-of-prosthetic-valves-a-case-series 